\title{
Detection of a large Be circumstellar disk during X-ray quiescence of XTE J1946+274
}

\author{
M. Özbey Arabac1 ${ }^{1}$, A. Camero-Arranz ${ }^{2}$, C. Zurita ${ }^{3,4}$, J. Gutiérrez-Soto ${ }^{5,6}$, E. Nespoli ${ }^{7}$, J. Suso ${ }^{7}$, F. Kiaeerad ${ }^{8,9}$, \\ J. García-Rojas ${ }^{3,4}$, and Ü. Kızıloğlu ${ }^{1}$ \\ 1 Department of Physics, Middle East Technical University, 06531 Ankara, Turkey \\ e-mail: mehtap@astroa.physics.metu.edu.tr \\ 2 Institut de Ciències de l'Espai, (IEEC-CSIC), Campus UAB, Fac. de Ciències, Torre C5 pa., 08193 Barcelona, Spain \\ 3 Instituto de Astrofísica de Canarias, 38200 La Laguna, Tenerife, Spain \\ ${ }^{4}$ Universidad de La Laguna, Dept. Astrofísica, 38206 La laguna, Tenerife, Spain \\ 5 Universitad de Valencia, Dept. Didáctica de las Matemática, Avda. Tarongers, 4, 46022 Valencia, Spain \\ ${ }^{6}$ Instituto de Astrofísica de Andalucía (CSIC), Glorieta de la Astronomía s/n, 18008 Granada, Spain \\ 7 Observatorio Astronómico de la Univ. de Valencia, C/Catedrático Jose Beltran, 2, 46980 Paterna (Valencia), Spain \\ 8 Nordic Optical Telescope, Apartado 474, 38700 Santa Cruz de La Palma, Spain \\ 9 Department of Astronomy, Oscar Klein Center, Stockholm University, AlbaNova, 10691 Stockholm, Sweden
}

Received 8 December 2014 / Accepted 9 August 2015

\section{ABSTRACT}

\begin{abstract}
Aims. We present a multiwavelength study of the Be/X-ray binary system XTE J1946+274 with the main goal of better characterizing its behavior during $\mathrm{X}$-ray quiescence. We also aim to shed light on the possible mechanisms which trigger the X-ray activity for this source.

Methods. XTE J1946+274 was observed by Chandra-ACIS during quiescence in 2013 March 12. In addition, this source has been monitored from the ground-based astronomical observatories of El Teide (Tenerife, Spain), Roque de los Muchachos (La Palma, Spain) and Sierra Nevada (Granada, Spain) since 2011 September, and from the TÜBİTAK National Observatory (Antalya, Turkey) since 2005 April. We have performed spectral and photometric temporal analyses in order to investigate the quiescent state and transient behavior of this binary system.

Results. Our optical study revealed that a long mass ejection event from the Be star took place in 2006, lasting for about seven years, and another one is currently ongoing. We also found that a large Be circumstellar disk is present during quiescence, although major $\mathrm{X}$-ray activity is not observed. We made an attempt to explain this by assuming the permanently presence of a tilted (sometimes warped) Be decretion disk. The 0.3-10 keV X-ray spectrum of the neutron star during quiescence was well fitted with either an absorbed black-body or an absorbed power-law models. The main parameters obtained for these models were $k T=1.43 \pm 0.17$ and $\Gamma=0.9 \pm 0.4$ (with $N_{\mathrm{H}} \sim 2-7 \times 10^{22} \mathrm{~cm}^{-2}$ ). The $0.3-10 \mathrm{keV}$ flux of the source was $\sim 0.8-1 \times 10^{-12} \mathrm{erg}^{-1} \mathrm{~cm}^{-2} \mathrm{~s}^{-1}$. Pulsations were found with $P_{\text {pulse }}=15.757(1)$ s (epoch MJD 56363.115) and an rms pulse fraction of 32.1(3)\%. The observed X-ray luminosity during quiescent periods was close to that of expected in supersonic propeller regimen.
\end{abstract}

Key words. accretion, accretion disks - stars: emission-line, Be - X-rays: binaries - pulsars: individual: XTE J1946+274

\section{Introduction}

The hard X-ray transient XTE J1946+274 is one of the poorlyunderstood sources among Be/X-ray binaries (BeXRB), although its X-ray behavior have been studied in detail since its discovery with All Sky Monitor (ASM) on board the Rossi X-Ray Timing Explorer (RXTE) in 1998 (Smith \& Takeshima 1998). The system showed two main transient X-ray active phases detected with different X-ray satellites between 1998 and 2011. The first and the longest X-ray activity lasted about $\sim 3 \mathrm{yr}$ (between September 1998-August 2001) including 13 consecutive outbursts (Wilson et al. 2003). During the initial outburst of these series, having the peak X-ray flux of $\sim 110 \mathrm{mCrab}$ in 2-60 keV band, it was revealed that the system had an X-ray pulsar with a spin period of $15.83 \pm 0.02 \mathrm{~s}$ (Smith \& Takeshima 1998; Wilson et al. 1998) orbiting around its Be companion with a period of 169.2 days in an 0.33 eccentric orbit (Campana et al. 1999; Wilson et al. 2003). Using optical and IR observations
Verrecchia et al. (2002) found a distance for XTE J1946+274 in the range of $8-10 \mathrm{kpc}$, in agreement with the value of $9.5 \pm 2.9 \mathrm{kpc}$ obtained from the X-ray data by Wilson et al. (2003). In addition, the existence of a cyclotron resonance scattering feature (CRSF) at $\sim 35 \mathrm{keV}$ was reported by Heindl et al. (2001) using the 1998 outburst observations of High Energy X-Ray Timing Experiment (HEXTE) and Proportional Counter Array (PCA) on RXTE.

After a $\sim 9$ yr quiescence in X-rays, the system underwent a new outburst phase starting on 2010 June 4 , reaching a value of $140 \mathrm{mCrab}$ in the $15-50 \mathrm{keV}$ energy band within 22 days on the Swift/Burst Alert Telescope (BAT) hard X-ray transient monitor (Krimm et al. 2010; Müller et al. 2012). Similar to the previous active phase of the source, the second outburst period was again in a series including an initial giant outburst followed by four fainter outbursts (Camero-Arranz et al. 2010b; Caballero et al. 2010; Nakajima et al. 2010; Müller et al. 2010). The presence of another CRSF at $\sim 25 \mathrm{keV}$ was discovered indicating 


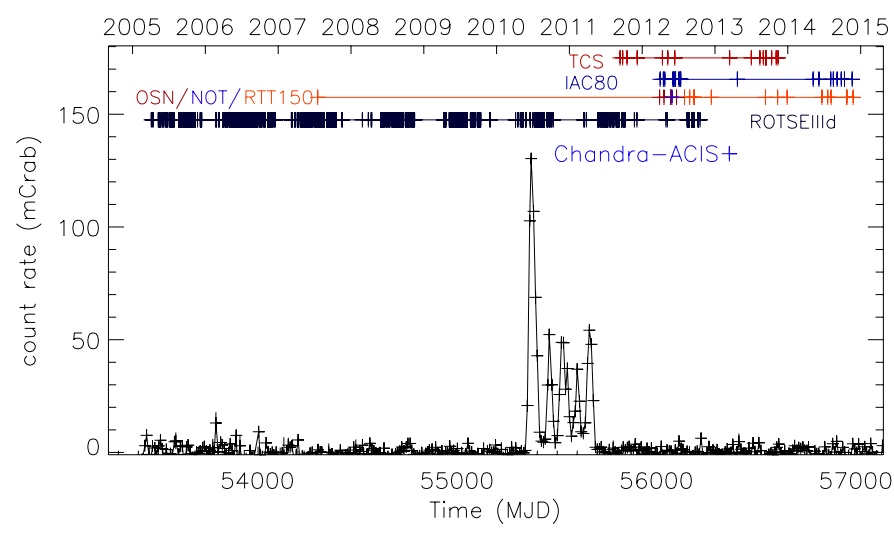

Fig. 1. Swift/BAT light-curve $(15-50 \mathrm{keV})$ with a bin size equal to $10 \mathrm{~d}$. Tick marks on the segments located above the light-curve denote the times of the Chandra pointing observation (light blue), as well as the optical/IR photometric data from the ground-based telescopes ROTSEIIId (black), IAC80 (dark blue) and TCS (dark red). The optical spectroscopic observations come from OSN (dark red), NOT (blue) and RTT150 (light red; see also Table 2).

Table 1. Log of the Chandra-ACIS observation during quiescence period II.

\begin{tabular}{ccccccc}
\hline \hline ObsID & $\begin{array}{l}\text { Date Obs. } \\
(\mathrm{UT})\end{array}$ & MJD & $\begin{array}{c}\text { Exp. } \\
(\mathrm{ks})\end{array}$ & $\begin{array}{c}\text { Rate } \\
(\mathrm{c} / \mathrm{s})\end{array}$ & $\begin{array}{c}P_{\text {pulse }} \\
(\mathrm{s})\end{array}$ & $\begin{array}{c}\mathrm{PF}_{\text {rms }}^{a} \\
(\%)\end{array}$ \\
\hline 14646 & $\begin{array}{c}2013-03-12 \\
02: 45: 36.00\end{array}$ & 56363.115 & 4.6 & $0.042(3)$ & $15.757(1)$ & $32.1(2)$ \\
& 02.36 & & & \\
\hline
\end{tabular}

Notes. ${ }^{(a)}$ Further details in Sect. 4.3.

the variation of cyclotron lines between the different outbursts (Müller et al. 2012).

In general, X-ray active phases of XTE J1946+274 include two outbursts per orbital period that are possible to be produced if the Be disk and the orbital plane are offset (Priedhorsky \& Holt 1987). However for XTE J1946+274 the outbursts do not coincide with the time of periastron and apastron passages of the neutron star (NS) that would be expected to happen for similar misaligned Be/X-ray systems (Wilson et al. 2003; Müller et al. 2012, and references therein). Therefore some other additional mechanisms would be responsible for this unique behavior.

The optical/IR counterpart to XTE J1946+274 was discovered by Verrecchia et al. (2002) nearly $\sim 3$ yr after the first X-ray activity of the system. It is a relatively faint $V=16.9$, reddened $\mathrm{B} 0-1 \mathrm{IV}-\mathrm{Ve}$ type star having strong $\mathrm{H} \alpha$ and $\mathrm{H} \beta$ emission lines in its spectra suggesting the presence of the decretion disk. Subsequent optical spectroscopic observations revealed the profile variations of $\mathrm{H} \alpha$ emission lines implying the existence of global density perturbations in the disk (Wilson et al. 2003).

In this work we present the results of a long-term multiwavelength campaign of XTE J1946+274. Since this X-ray transient spends most of its life in X-ray quiescent phase, our spectroscopic and photometric data mainly cover these inactive periods between the X-ray brightening phases. These optical/IR data come from our monitoring program on $\mathrm{Be} / \mathrm{X}$-ray binaries, which involves several ground-based astronomical observatories. In addition, we used one Chandra-ACIS pointed observation carried out during X-ray quiescence in 2013, and several survey data from different space-borne telescopes to investigate the relation between the X-ray and optical/IR bands.
Table 2. H $\alpha$ equivalent width (EW) measurements of optical counterpart to XTEJ1946+274.

\begin{tabular}{lllrc}
\hline \hline Date & MJD & $E W(\AA)$ & $F W H M(\AA)$ & Telescope \\
\hline 2007-Jul.-18 & 54299.816 & $-37.35 \pm 1.39$ & $9.88 \pm 0.27$ & RTT150 \\
2012-Mar.-27 & 56013.148 & $-17.73 \pm 0.76$ & $11.09 \pm 0.46$ & OSN \\
2012-Apr.-18 & 56035.132 & $-28.49 \pm 1.20$ & $11.57 \pm 0.30$ & OSN \\
2012-May-22 & 56069.014 & $-41.65 \pm 1.31$ & $10.19 \pm 0.46$ & OSN \\
2012-May-22 & 56069.039 & $-40.70 \pm 1.39$ & $9.87 \pm 0.15$ & OSN \\
2012-May-28 & 56075.024 & $-45.16 \pm 1.36$ & $10.40 \pm 0.78$ & NOT \\
2012-Jun.-19 & 56097.997 & $-39.87 \pm 1.52$ & $10.30 \pm 0.11$ & OSN \\
2012-Jul.-4 & 56112.963 & $-45.07 \pm 1.01$ & $9.69 \pm 0.74$ & OSN \\
2012-Jul.-5 & 56113.030 & $-47.62 \pm 0.95$ & $10.21 \pm 0.94$ & OSN \\
2012-Jul.-29 & 56137.922 & $-39.12 \pm 0.86$ & $10.12 \pm 0.32$ & RTT150 \\
2012-Aug.-11 & 56150.981 & $-44.46 \pm 1.18$ & $10.62 \pm 0.44$ & OSN \\
2012-Aug.-24 & 56163.847 & $-41.64 \pm 1.74$ & $10.14 \pm 0.31$ & RTT150 \\
2012-Sep.-15 & 56185.758 & $-40.00 \pm 0.86$ & $9.78 \pm 0.31$ & RTT150 \\
2012-Sep.-16 & 56186.734 & $-39.65 \pm 1.15$ & $9.76 \pm 0.31$ & RTT150 \\
2012-Oct.-09 & 56209.917 & $-42.18 \pm 1.11$ & $11.66 \pm 0.26$ & OSN \\
2012-Dec.-11 & 56272.669 & $-42.58 \pm 2.04$ & $10.35 \pm 0.31$ & RTT150 \\
2013-Sep.-08 & 56543.815 & $-40.11 \pm 1.35$ & $9.88 \pm 0.31$ & RTT150 \\
2013-Nov.-08 & 56604.714 & $-36.11 \pm 0.58$ & $13.33 \pm 0.13$ & RTT150 \\
2013-Dec.-26 & 56652.890 & $-38.68 \pm 1.79$ & $14.09 \pm 0.88$ & RTT150 \\
2014-Jun.-19 & 56827.041 & $-39.60 \pm 0.97$ & $9.07 \pm 0.49$ & RTT150 \\
2014-Jul.-17 & 56855.813 & $-42.79 \pm 1.10$ & $9.95 \pm 0.60$ & RTT150 \\
2014-Aug.-03 & 56872.859 & $-41.06 \pm 0.80$ & $9.85 \pm 0.43$ & RTT150 \\
2014-Oct.-20 & 56950.753 & $-43.14 \pm 1.28$ & $9.83 \pm 0.49$ & RTT150 \\
2014-Oct.-21 & 56951.774 & $-42.37 \pm 1.15$ & $9.99 \pm 0.45$ & RTT150 \\
2014-Nov.-22 & 56983.714 & $-38.24 \pm 1.12$ & $10.00 \pm 0.47$ & RTT150 \\
\hline & & & &
\end{tabular}

\section{Observations and data reduction}

\subsection{Optical spectroscopic observations}

Optical spectroscopic observations of the companion were performed during 2011 April-2014 November with four different telescopes: the Russian-Turkish 1.5-m telescope (RTT150) at the TÜBITAK National Observatory in Antalya (Turkey), the 2.56-m Nordic Optical Telescope (NOT) located at the Observatorio del Roque de los Muchachos (La Palma, Spain), and the 1.5-m Telescope at the Observatorio de Sierra Nevada (OSN-CSIC) in Granada (Spain). In addition to this long-term observation set we include a spectrum of the source taken in June 2007 with RTT150.

The spectroscopic data from RTT150 were obtained with the TÜBITTAK Faint Object Spectrometer and Camera (TFOSC). It is equipped with a $2048 \times 2048,15 \mu \mathrm{m}$ pixel Fairchild 447BI CCD whose FOV is $13.3 \times 13 ! 3$. We used slit $67 \mu \mathrm{m}\left(1{ }^{\prime \prime} 24\right)$ with Grism 8 having an average dispersion of $1.1 \AA$ pixel and providing a 5800-8300 $\AA$ wavelength coverage. The reduction of RTT150 spectra was done using the Long-Slit package of MIDAS $^{1}$. Bias correction, flat-fielding and removal of cosmicray hits were carried out with standard MIDAS routines.

The low-resolution OSN spectra $(R \approx 1400)$ were acquired using Albireo spectrograph centred on $\mathrm{H} \alpha$ wavelength (6562.8 $\AA$ ) whereas NOT spectrum was obtained with the Andalucía Faint Object Spectrograph and Camera (ALFOSC) ${ }^{2}$, using Grism 7, with a dispersion of $1.5 \AA /$ pixel, and 0.'5-1" slits. The reduction of this data set was performed using standard

1 http://www.eso.org/projects/esomidas

2 The data presented here were obtained (in part) with ALFOSC, which is provided by the Instituto de Astrofísica de Andalucía (IAA) under a joint agreement with the University of Copenhagen and NOTSA. 
Table 3. Optical and IR magnitudes of XTE J1946+274 observed with IAC 80 and TCS telescopes respectively.

\begin{tabular}{|c|c|c|c|c|c|c|}
\hline Date & MJD & $B$ & $V$ & $J$ & $H$ & $K_{\mathrm{s}}$ \\
\hline 2011-Sep.-11 & 55815.029 & & & $12.450 \pm 0.028$ & - & - \\
\hline 2011-Sep.-23 & 55827.959 & - & - & $12.694 \pm 0.059$ & $11.727 \pm 0.048$ & - \\
\hline 2011-Sep.-24 & 55828.962 & - & - & - & - & $11.402 \pm 0.066$ \\
\hline 2011-Oct.-15 & 55849.970 & - & - & $12.562 \pm 0.000$ & $11.789 \pm 0.019$ & $10.520 \pm 0.088$ \\
\hline 2011-Dec.-05 & 55900.815 & - & - & $12.602 \pm 0.042$ & $11.899 \pm 0.152$ & $11.260 \pm 0.055$ \\
\hline 2012-Mar.-30 & 56016.195 & $18.737 \pm 0.056$ & $15.784 \pm 0.050$ & - & - & - \\
\hline 2012-Apr.-10 & 56027.140 & & & $12.560 \pm 0.073$ & $11.828 \pm 0.009$ & $11.240 \pm 0.105$ \\
\hline 2012-Apr.-15 & 56032.192 & $18.803 \pm 0.062$ & $15.89 \pm 0.051$ & - & - & \\
\hline 2012-Apr.-22 & 56039.195 & - & $15.898 \pm 0.051$ & - & - & \\
\hline 2012-Мay-07 & 56054.179 & - & - & $12.734 \pm 0.083$ & $11.856 \pm 0.019$ & $11.326 \pm 0.136$ \\
\hline 2012-Jun.-02 & 56080.183 & $18.788 \pm 0.055$ & $15.835 \pm 0.050$ & - & - & - \\
\hline 2012-Jun.-04 & 56082.206 & $18.627 \pm 0.074$ & $15.784 \pm 0.051$ & - & - & - \\
\hline 2012-Jun.-11 & 56089.214 & $18.822 \pm 0.069$ & $15.793 \pm 0.050$ & - & - & - \\
\hline 2012-Jun.-14 & 56092.957 & $18.708 \pm 0.063$ & $15.841 \pm 0.051$ & - & - & - \\
\hline 2010-Jul.-01 & 56109.913 & - & $15.773 \pm 0.054$ & - & - & - \\
\hline 2012-Jul.-07 & 56115.892 & $18.863 \pm 0.088$ & $15.777 \pm 0.056$ & - & - & - \\
\hline 2012-Jul.-10 & 56118.885 & $18.726 \pm 0.087$ & $15.795 \pm 0.051$ & - & - & - \\
\hline 2013-Mar.-13 & 56364.225 & - & - & $12.504 \pm 0.015$ & $11.897 \pm 0.025$ & $11.308 \pm 0.020$ \\
\hline 2013-Apr.-21 & 56403.164 & $18.582 \pm 0.042$ & $15.666 \pm 0.026$ & - & - & - \\
\hline 2013-Apr.-22 & 56404.145 & - & - & $12.591 \pm 0.007$ & - & $11.278 \pm 0.060$ \\
\hline 2013-Мау-03 & 56415.075 & - & - & $12.446 \pm 0.057$ & - & $11.202 \pm 0.084$ \\
\hline 2013-Jun.-30 & 56473.074 & - & - & $12.325 \pm 0.006$ & $11.727 \pm 0.071$ & $11.227 \pm 0.059$ \\
\hline 2013-Jul.-12 & 56485.011 & - & - & $12.464 \pm 0.016$ & - & - \\
\hline 2013-Aug.-11 & 56515.996 & - & - & $12.493 \pm 0.022$ & $11.262 \pm 0.068$ & - \\
\hline 2013-Aug.-28 & 56532.892 & - & - & $12.517 \pm 0.020$ & $11.817 \pm 0.009$ & $11.255 \pm 0.037$ \\
\hline 2013-Sep.-09 & 56544.937 & - & - & $12.424 \pm 0.006$ & $11.688 \pm 0.005$ & $11.196 \pm 0.023$ \\
\hline 2013-Sep.-11 & 56546.022 & - & - & $12.324 \pm 0.049$ & $11.745 \pm 0.009$ & $11.209 \pm 0.054$ \\
\hline 2013-Sep.-11 & 56546.942 & - & - & $12.463 \pm 0.031$ & $11.845 \pm 0.009$ & $11.278 \pm 0.064$ \\
\hline 2013-Oct.-09 & 56574.887 & - & - & - & $11.799 \pm 0.044$ & $11.286 \pm 0.063$ \\
\hline 2013-Nov.-01 & 56597.927 & - & - & $12.493 \pm 0.035$ & $11.797 \pm 0.007$ & $11.269 \pm 0.095$ \\
\hline 2013-Nov.-10 & 56606.872 & - & - & $12.455 \pm 0.070$ & $11.826 \pm 0.090$ & $11.294 \pm 0.007$ \\
\hline 2014-Мау-06 & 56783.146 & $18.582 \pm 0.047$ & $15.683 \pm 0.051$ & - & - & - \\
\hline 2014-Jun.-04 & 56812.110 & $18.470 \pm 0.058$ & $15.591 \pm 0.054$ & - & - & - \\
\hline 2014-Aug.-02 & 56871.111 & $18.416 \pm 0.043$ & $15.565 \pm 0.051$ & - & - & - \\
\hline 2014-Aug.-15 & 56884.073 & $18.503 \pm 0.049$ & $15.489 \pm 0.051$ & - & - & - \\
\hline 2014-Sep.-03 & 56903.022 & $18.378 \pm 0.044$ & $15.537 \pm 0.051$ & - & - & - \\
\hline 2014-Sep.-03 & 56903.026 & $18.433 \pm 0.045$ & - & - & - & - \\
\hline 2014-Sep.-23 & 56923.926 & $18.441 \pm 0.042$ & $15.540 \pm 0.051$ & - & - & - \\
\hline 2014-Oct.-10 & 56940.007 & $18.272 \pm 0.094$ & $15.501 \pm 0.054$ & - & - & - \\
\hline 2014-Nov.-17 & 56978.825 & $18.558 \pm 0.042$ & $15.646 \pm 0.051$ & - & - & - \\
\hline
\end{tabular}

procedures within $\mathrm{IRAF}^{3}$, including bias subtraction, removal of pixel-to-pixel sensitivity variations, optimal spectral extraction, and wavelength calibration based on arc-lamp spectra.

All spectroscopic data were normalized with a spline fit to continuum and corrected to the barycenter after the wavelength calibration. The full width at half maximum (FWHM) and EW measurements of $\mathrm{H} \alpha$ lines were acquired by fitting Gaussian functions to the emission profiles using the ALICE subroutine of MIDAS.

\subsection{Optical/IR photometric observations}

As a part of our monitoring campaign the optical counterpart to XTEJ1946+274 has been observed in the optical and infrared bands during the period of 2011 September-2014 November (see Table 3) with the $80-\mathrm{cm}$ IAC80 and the $1.5-\mathrm{m}$ TCS telescopes at the Observatorio del Teide on Tenerife (Spain)

\footnotetext{
3 IRAF is distributed by the National Optical Astronomy Observatory, optical images which is operated by the Association of Universities for Research in Astronomy (AURA) under cooperative agreement with the National Science Foundation.
}

respectively. We obtained the optical photometric CCD images using $B$ and $V$ filters with integration time of 120 s. In infrared, $J, H$ and $K_{\mathrm{s}}$ simultaneous observations were performed using the CAIN camera with integration times of $150 \mathrm{~s}$. The reduction of the data was done by using the pipelines of both telescopes based on the standard aperture photometry (Camero et al. 2014, for more details on reduction).

The main part of the long-term optical CCD observations of the source include the $0.45-\mathrm{m}$ reflecting ROTSEIIId ${ }^{4}$ telescope data achieved from 2005 April to 2012 November (MJD 53 465-56215). ROTSEIIId telescope, located at the TÜBITTAK National Observatory (Antalya, Turkey), operates without filters is equipped with a $2048 \times 2048$ pixel CCD. For a total field of view (FOV) $1.85 \times 1.85$, the pixel scale is defined as 3.' 3 pixel $^{-1}$ (Akerlof et al. 2003). During the observations, a total of 2014 CCD frames were collected covering almost 8 seasons. Dark and flat-field corrections of all images were done automatically by a pipeline immediately after the pointing.

4 The Robotic Optical Transient Search Experiment, ROTSE, is a collaboration of Lawrence Livermore National Lab, Los Alamos National Lab, and the University of Michigan (http://www. ROTSE . net). 
Instrumental magnitudes of all the corrected images were obtained using an aperture of 3 pixels $(10 \mathrm{arcsec})$ in diameter by SExtractor Package (Bertin \& Arnouts 1996). By comparing all the stars in each frames with USNO-A2.0 catalog $R$-band magnitudes, calibrated ROTSEIIId magnitudes were acquired. For the timing analysis the time series were corrected to the barycenter by using JPL DE200 ephemerides (K1zıloğlu et al. 2005, for the details of ROTSEIIId data reduction).

\subsection{X-ray observations}

The Chandra X-ray Observatory observed XTE J1946+274 with the Advanced CCD Imaging Spectrometer (ACIS) instrument in FAINT mode in 2013 March 12 (MJD 56363.115) for a total exposure time of $4.6 \mathrm{ks}$. Figure 1 and Table 1 provide the $\log$ of this observation. We used a $1 / 8$ subarray, which provides a time resolution of $0.4 \mathrm{~s}$, and the typical ACIS-S imaging and spectral configurations. The source was positioned in the back-illuminated ACIS-S3 CCD at the nominal target position. Standard processing of the data was performed by the Chandra X-ray Center (CXC) to Level 1 and Level 2 (processing software DS ver. 8.5.1.1). In this work we have used CIAO software (ver. 4.6) for the reprocessing and the analysis of the data. Since 2008, the Gamma-ray Burst Monitor (GBM) on board the Fermi satellite, has been monitoring XTE J1946+274. In this study we used timing products provided by the GBM Pulsar Team (see e.g. Finger et al. 2009; Camero-Arranz et al. 2010a, for a detailed description of the timing technique). We also used quick-look X-ray results provided by the RXTE All Sky Monitor team ${ }^{5}$, and Swift/BAT transient monitor results provided by the Swift/BAT team (Krimm et al. 2013).

\section{Analysis and results}

\subsection{Optical/IR photometry}

The results of our multiwavelength campaign are shown in Fig. 2. In this figure the optical/IR behavior of the Be star is displayed, together with the X-ray activity observed from the NS (see Table 3). The ROTSE magnitude (fourth panel, from top to bottom) steadily varied from 2005 to 2013, with a minimum in 2006 and a maximum around mid 2010. A main X-ray outburst was then detected in 2010 lasting $~ 60$ days and reaching a flux of $\sim 140 \mathrm{mCrab}$ in the $15-50 \mathrm{keV}$ energy range (see bottom panel of Fig 2). The following four outbursts were seen in a series, typical to Type I outbursts, with a separation of 60-90 days. In contrast to the other BeXRB systems showing recurrent series of normal outbursts, they did not coincide with the time of periastron passage of the NS. After the 2010/2011 X-ray outburst series, the Be/X-ray binary XTE J1946+274 did not show any transient activity. The quiescent phase has been ongoing for $\sim 4 \mathrm{yr}$, although the optical/IR magnitudes have been showing an increasing trend since 2012 (see second and third panels of the same figure).

In order to search for periodic variability in the daily ROTSE light curve we used the Lomb-Scargle (Scargle 1982) and Clean (Roberts et al. 1987) algorithms. The frequency analysis was applied to all photometric data, including 1747 points, over a range from 0 to $17.49 \mathrm{~d}^{-1}$ (Nyquist frequency). We did not find any periodic variation in the original data, except for the power at the frequency of $1 \mathrm{~d}^{-1}$, as a result of the daily observing schedule. We then rebinned the light curve in 30 days bins. Figure 3

\footnotetext{
5 http://xte.mit.edu/ASM_lc.html
}

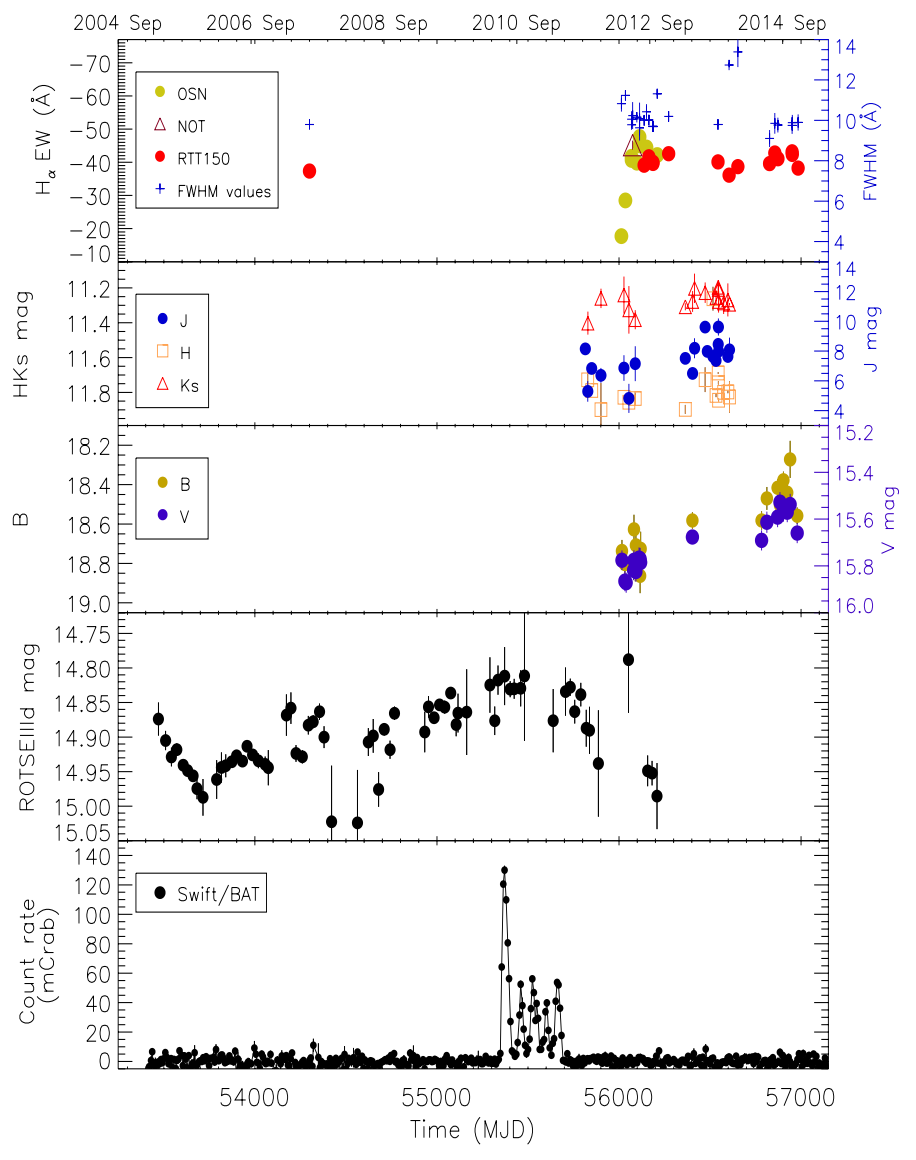

Fig. 2. Optical/IR and X-ray evolution of XTE J1946+274. The time bin for the ROTSEIIId lightcurve is 30 days and for Swift/BAT is 8 days.

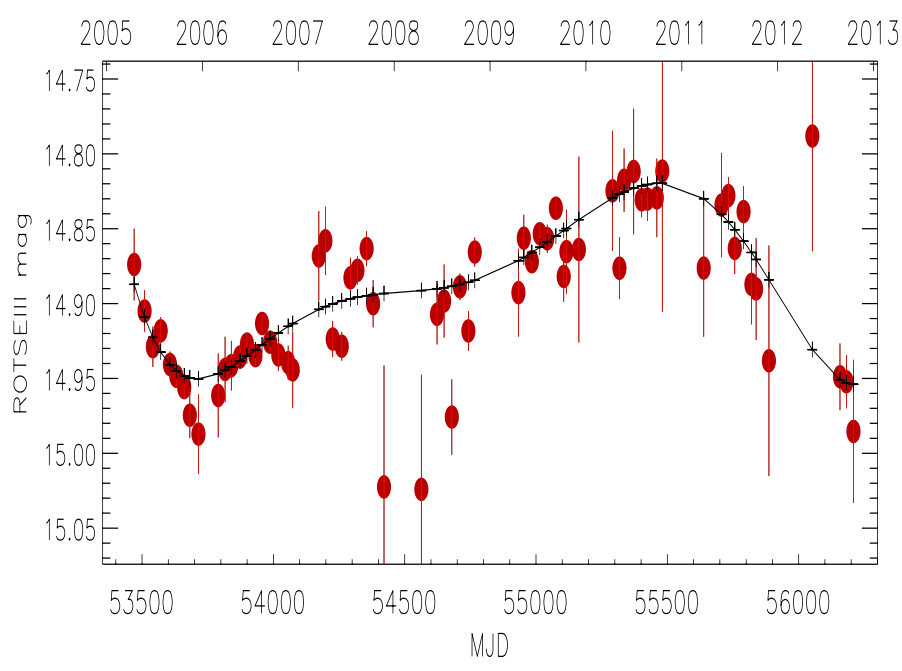

Fig. 3. Complete ROTSEIIId light curve binned using 30 days bins. Overplotted is the fitted sinusoid to the data (see Sect. 3 for more details).

reveals a quasi-sinusoidal pattern in the data, with a minimum at the beginning of 2006 (MJD 53700). This seems to indicate that a brightening phase of the Be star occurred for almost five years, followed by a quick decline in about 2 yr. To clarify this point we used the Fourier analysis module included in the PeriodQ4 software package ${ }^{6}$ (Lenz \& Breger 2005), which is based on a discrete Fourier transform algorithm. We thus fitted

6 http://www . astro.univie.ac.at/dsn/dsn/Period04 

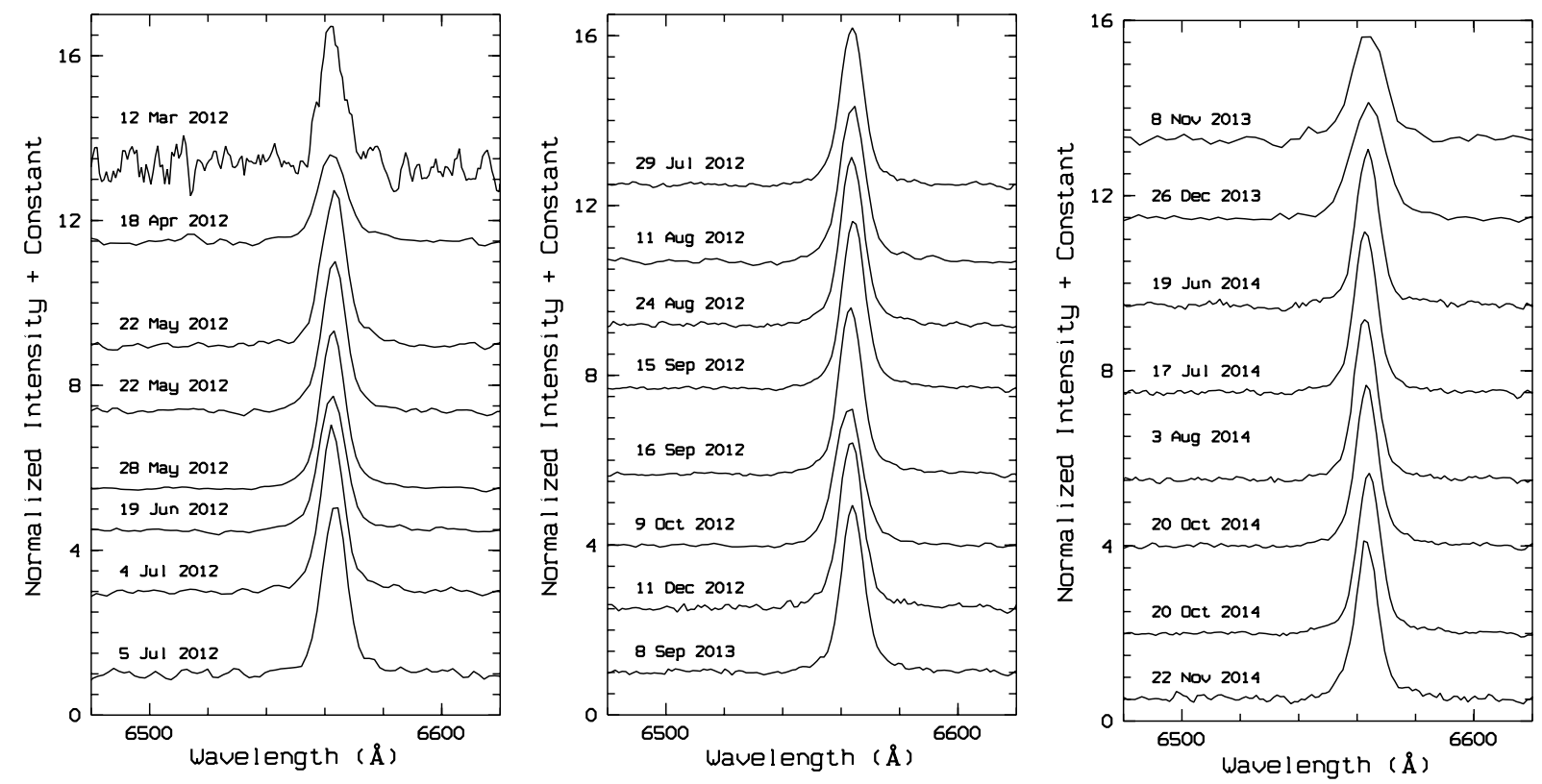

Fig. 4. $\mathrm{H} \alpha$ line profile evolution during the $\mathrm{X}$-ray quiescence. The presence of any remarkable variation is not detected.

a sinusoid to a combination of the observed frequency $3.3(8) \times$ $10^{-4} \mathrm{~d}^{-1}(\sim 3030 \mathrm{~d})$, found by PeriodQ4, together with its first and second harmonics. The uncertainties in the frequency determinations were computed from Monte Carlo simulations. The resulted fitted curve (see also Fig. 3) shows the probable evolution of the Be star brightening episode, probably a mass ejection event.

\section{2. $\mathrm{H} \alpha$ line}

In Fig. 4, we present the spectroscopic tracing of the $\mathrm{H} \alpha$ line profiles observed between 2012-2014. The H $\alpha$ line was always seen in a single-peaked emission (as the one in 2007 July 18) whereas EW and FWHM measurements showed significant variations (see top panel of Fig. 2). The first spectroscopic observation of this period had the lowest value of $\mathrm{H} \alpha$ emission line, $\sim 18 \AA$, ever observed for XTEJ1946+274. The weakness of this emission comparing to the typical values of XTE J1946+274 might be interpreted as the variations in the decretion disk of the Be star. Assuming that the EW measurement of $\mathrm{H} \alpha$ line emission was also related to the amount of the material in the emitting region of the disk, the weakness of the EW would be the result of the mass loss either through the accretion of the NS or the truncation of its size despite the lack of an X-ray activity. It is also possible that the weakest value we caught does not represent the lowest one, instead it can be a part of the refilling process of the disk after a disk-loss episode. In fact, a sharp increasing trend of the EW right after this value confirms the suggested idea. The increase in EW lasted about three months reaching its peak value of $\sim 48 \AA$. It is also important to note that this is the highest EW value of XTE J1946+274 ever observed. Although the line measurements were scattered between MJD 56069-56209, they were not significantly different than the average EW value.

During the observations of October and December 2013, the widest emissions were detected while EW values were around the average (see top panel of Fig. 2). In general the $\mathrm{H} \alpha$ line was seen as a narrow single-peaked emission with an average FWHM value of $\sim 10.5 \AA$. It is also important to note that the
EW and FWHM values of the source show an inverse relation despite the expected positive relation (Hanuschik 1989).

In addition, we computed the rotational velocity of the Be star. First we estimated the projected rotational velocity of XTE J1946+274 as $v \sin i \sim 323 \mathrm{~km} \mathrm{~s}^{-1}$ via the average values of EW and FWHM parameters (Hanuschik 1989). Then using the inclination angle of the system given by Wilson et al. (2003), the true rotational velocity, $v_{\text {rot }}$, of the Be star was measured as $323-449 \mathrm{~km} \mathrm{~s}^{-1}$. Taking the mass of the star as $16 M_{\odot}$ and $8 R_{\odot}$ as the limit radius for a $\mathrm{B}$ type star, then the critical breakup velocity, $v_{\text {crit }}$, was found to be $\sim 618 \mathrm{~km} \mathrm{~s}^{-1}$. Thus, we find the critical fraction, defined as the the ratio of the equatorial rotational velocity to the the break-up velocity, of XTE J1946+274 as $w \sim 0.5-0.72$. This result indicates that the Be star in XTE J1946+274 is rotating at 50-70\% of its break-up velocity, typical to the stars for the same type.

\section{Chandra/ACIS-S X-ray analysis during quiescence}

\subsection{Imaging}

We extracted an image in the $0.3-10 \mathrm{keV}$ energy range, using the Chandra pointing observation from 2013 as described in Sect. 2.3. We then applied the CIAO celldetect tool to the $\sim 4.6 \mathrm{ks}$ ACIS-S cleaned image and found XTE J1946+274 at $\alpha=19^{\mathrm{h}} 45^{\mathrm{m}} 39^{\mathrm{s}} .34$ and $\delta=27^{\circ} 21^{\prime} 55^{\prime \prime} .36$ (J2000) with a signalto-noise ratio of 11.025 , and a statistical error of 0.03 arcsec radius. In addition, another $\mathrm{X}$-ray bright star was detected at $\alpha=19^{\mathrm{h}} 45^{\mathrm{m}} 34^{\mathrm{s}} .91$ and $\delta=27^{\circ} 18^{\prime} 18^{\prime \prime}$. 04 with a signal-to-noise ratio of 5.2, and a statistical error of 0.1 arcsec radius.

Furthermore, XTEJ1946+274 is the 2MASS $19453935+2721555$ star with a catalog position of $\alpha=$ $19^{\mathrm{h}} 45^{\mathrm{m}} 39^{\mathrm{s}} .36$ and $\delta=27^{\circ} 21^{\prime} 55^{\prime \prime} .52$ (J2000) (statistical error of 0.06 arcsec radius) and the second star is the 2MASS $19453491+2718183$ with a catalog position of $\alpha=19^{\mathrm{h}} 45^{\mathrm{m}} 34.92$ and $\delta=27^{\circ} 18^{\prime} 18^{\prime \prime} .30$ (0.06 arcsec error radius). We then performed a boresight correction of the field to refine XTEJ1946+274 position and its error circle. 
Table 4. Spectral parameters from an absorbed PL model and absorbed BB.

\begin{tabular}{lll}
\hline \hline Parameter & PL & BB \\
\hline$N_{\mathrm{H}}{ }^{a}$ & $0.7(4)$ & $0.21(15)$ \\
$\Gamma$ & $0.9(4)$ & - \\
$\Gamma_{\text {norm }}{ }^{b}$ & $1.03(3)$ & - \\
$k T(\mathrm{keV})$ & - & $1.43(17)$ \\
$k T_{\text {norm }}$ & - & $0.020(8)$ \\
abs.Flux $^{c}$ & $1.0(3)$ & $0.81(16)$ \\
unabs.Flux $^{c}$ & $1.2(4)$ & $0.84(18)$ \\
C-stat (d.o.f.) & $108.32(148)$ & $109.15(148)$ \\
\hline
\end{tabular}

Notes. Errors are given at the $90 \%$ confidence level. ${ }^{(a)} \times 10^{22} \mathrm{~cm}^{-2}$. (b) $\times 10^{-4}$ photons $\mathrm{keV}^{-1} \mathrm{~cm}^{-2} \mathrm{~s}^{-1}$ at $1 \mathrm{keV}$. ${ }^{(c)} \times 10^{-12} \mathrm{erg} \mathrm{cm}^{-2} \mathrm{~s}^{-1}$ in the $0.3-10 \mathrm{keV}$ energy band.

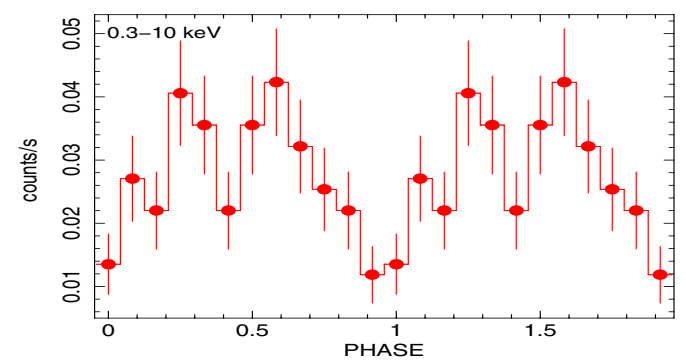

Fig. 5. Background subtracted X-ray pulse profile (in counts $\mathrm{s}^{-1}$ ) for XTE J1946+274 during quiescence in the $0.3-10 \mathrm{keV}$ energy band.

Assuming a physical association between the 2MASS stars and the X-ray sources, the final position of XTEJ1946+274 is $\alpha=19^{\mathrm{h}} 45^{\mathrm{m}} 39^{\mathrm{s}} .4$ and $\delta=27^{\circ} 21^{\prime} 55^{\prime \prime}$. 5 with a $1 \sigma$ associated error circle of 0.2 arcsec radius (computed doing a quadratic mean of all the positional and statistical errors plus the 2MASS catalogue intrinsic systematic errors).

\subsection{Spectral study}

To obtain the $0.3-10 \mathrm{keV}$ phase-averaged spectrum for the Chandra ACIS-S observation we used source and background photons extracted as described in Sect. 2. We used the specextract script, which uses a combination of CIAO tools, to extract source and background spectra for XTE J1946+274. To extract only the photons from the point source a circular region with 2 .'5 radius and a circular background region of radii $18^{\prime \prime}$ (far from the source) were used. For the present analysis we used the XSPEC package (version 12.8.1g; Arnaud 1996).

Two models provided the best fit to the data, an absorbed power law (PL) and blackbody (BB) models. For the photoelectric absorption we used the cross-sections from BalucinskaChurch \& McCammon (1992) and the Solar abundance from Anders \& Grevesse (1989). The best-fit parameters for these models can be seen in Table 4 . The main parameters for both models are $\Gamma=0.9 \pm 0.4$ (C-stat $=108.32$ for 148 d.o.f. $)$, and $k T=1.43 \pm 0.17 \mathrm{keV}$ (C-stat $=109.15$ for 148 d.o.f. $)$, with $N_{\mathrm{H}} \sim 2-7 \times 10^{22} \mathrm{~cm}^{-2}$. The $2-10 \mathrm{keV}$ observed flux was $F_{\mathrm{X}}=$ $0.75(3)-0.97(2) \times 10^{-12} \mathrm{erg} \mathrm{cm}^{-2} \mathrm{~s}^{-1}$ for the BB and PL models, respectively. The photon index is similar to the value found with Swift/XRT (Müller et al. 2012) in the 1.5-7 keV energy range $(\Gamma=0.84(8))$, and Suzaku-XIS in the $0.3-10 \mathrm{keV}$ band $(\Gamma=1.09(5))$. However, the X-ray flux level of XTE J1946+274 in those observations was about 2 order of magnitude larger $\left(\sim 2 \times 10^{-10} \mathrm{erg} \mathrm{cm}^{-2} \mathrm{~s}^{-1}\right)$.

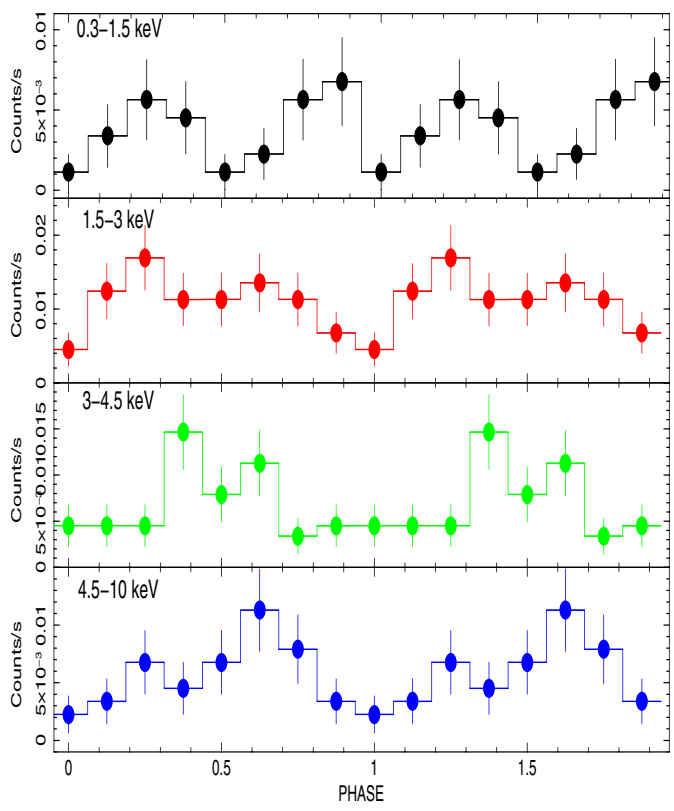

Fig. 6. Background subtracted X-ray pulse profiles (in counts/s) during quiescence in different energy bands.

\subsection{Timing}

For the timing analysis, we first referred the arrival time of each photon to the barycenter of the solar system using the CIAO tool axbary. Then, we used the dmextract tool to create background-subtracted lightcurves, using the time resolution of the data $(\sim 0.4 \mathrm{~s})$. For this, we extracted the source photons on each individual observation from a circular region with 2 .' 5 radius, and another one for the background, far from the source.

We searched for pulsations using the Xronos package and found a $P_{\text {pulse }}=15.757(1) \mathrm{s}($ epoch $=$ MJD 56363.115; $v=$ $0.063464(4) \mathrm{Hz}$ ). Figure 5 shows the pulse profile obtained by folding the X-ray data set using this period. Despite the low luminosity level of the source in this observation, we can observe a profile with two peaks separated by a dip at phase $\sim 0.4$, and another dip at phase 0.95 . Wilson et al. (2003) found that at its lowest intensity level during the 2001 outburst the profile consisted of an asymmetric structured main peak near phase 0.2 in an RXTE/PCA observation of XTE J1946+274. We note, however, that this profile was obtained in the $2-30 \mathrm{keV}$ energy range. In our Chandra/ACIS-S observation the dip in the pulse profile was more prominent at low energies in the $0.3-1.5 \mathrm{keV}$ band, vanishing as the energy increases (see Fig. 6), and with the pulse profile evolving to single-peaked. The shape of the profile at 3-4.5 keV was narrower and quite symmetric comparing to the asymmetric single peaks found at $1.5-3.5 \mathrm{keV}$ and $4.5-10 \mathrm{keV}$, with all peaking at different phases of $\sim 0.2, \sim 0.4$, and $\sim 0.6$ for each energy band.

The modulation amplitude of the $0.3-10 \mathrm{keV}$ pulse displayed in Fig. 5 (12 phase bins) can be measured using a pulse fraction defined as follows,

$P F_{\mathrm{rms}}=\frac{1}{\bar{y}} \sqrt{\frac{1}{n} \sum_{i=1}^{n}\left(\left(y_{i}-\bar{y}\right)^{2}-\sigma_{i}^{2}\right)}$

where $n$ is the number of phase bins per cycle, $y_{i}$ is the number of counts in the $i$ th phase bin, $\sigma_{i}$ is the error on $y_{i}$ and $\bar{y}$ is the mean number of counts in the cycle. Applying this formula to 


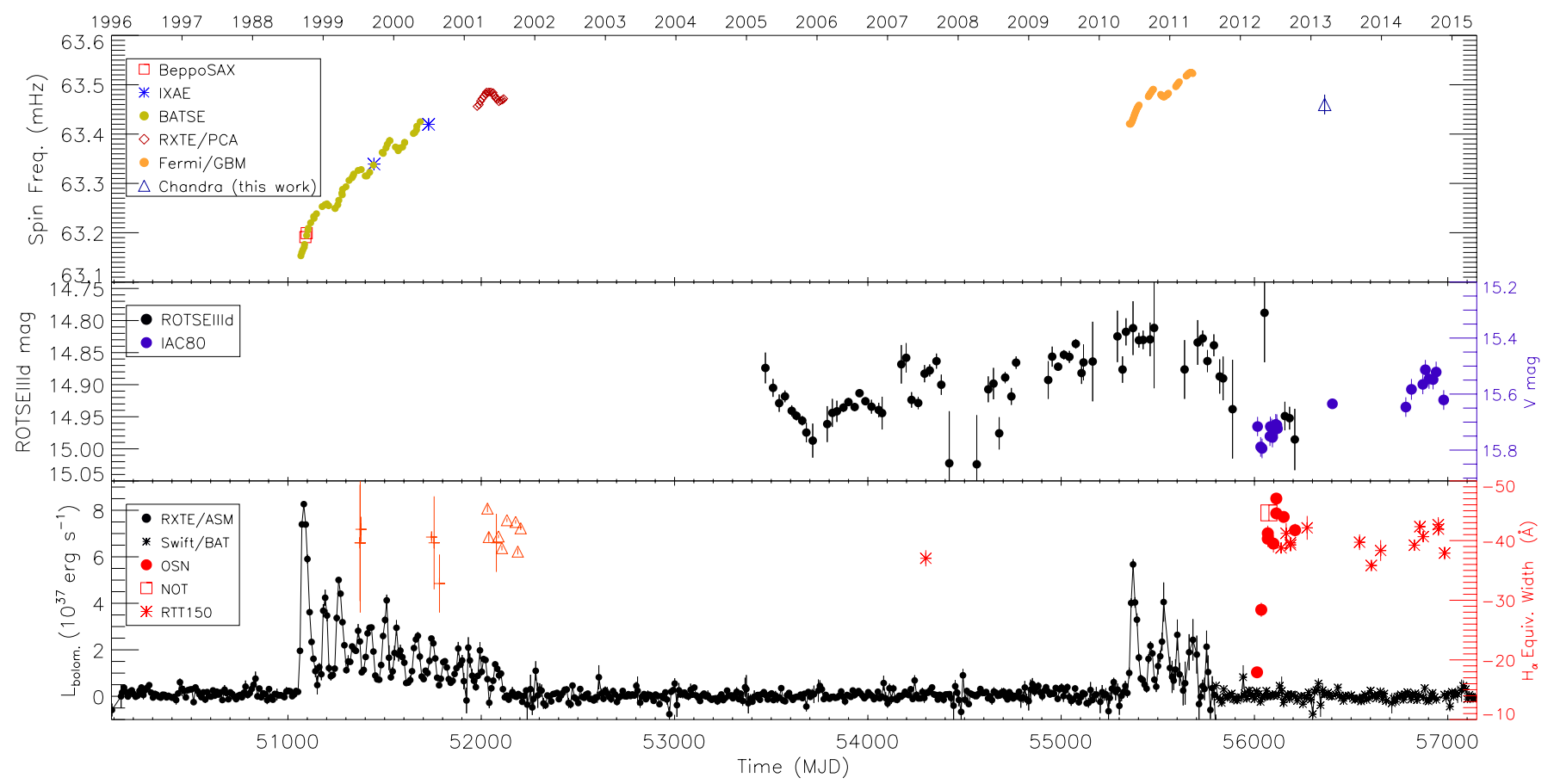

Fig. 7. Top: spin frequency history of XTE J1946+274 since its discovery in 1988. Middle: long-term optical light curve of this source. Bottom: long-term X-ray bolometric luminosity, and overplotted the evolution of the EW of the $\mathrm{H} \alpha$ line (red stars, open square, and filled circles). The $\mathrm{H} \alpha$ EW measurements from 1999 to 2002 were extracted from (Verrecchia et al. 2002, red crosses) and Wilson et al. (2003, red triangles). To compute the X-ray bolometric luminosity we follow the procedure by Wilson et al. (2003).

our data we obtained a $P F_{\text {rms }}=32.1(3) \%$. Using the standard definition,

$P F_{\text {std }}=\frac{F_{\max }-F_{\min }}{F_{\max }+F_{\min }}$

the value we obtained was larger, as expected, being $56(20) \%$. In addition, we found $P F_{\text {rms }}$ estimates of 51.02(12)\%, 18.52(2)\%, $54.27(13) \%$, and $37.23(15) \%$ in the $0.3-1.5 \mathrm{keV}, 1.5-3 \mathrm{keV}$, $3-4.5 \mathrm{keV}$, and $4.5-10 \mathrm{keV}$ energy bands, respectively. We would like to note that for the profiles at different energy bands the phase binning was reduced to 8 , since the $P F_{\text {rms }}$ formulation did not yield real solutions in some of the bands.

\section{Discussion}

\subsection{Be/NS interaction}

XTEJ1946+274 is one of the BeXRBs that spends most of its time in an X-ray quiescent phase. The uniqueness of the system comes from its X-ray outburst behavior that is not connected to the orbital passages of the NS. The shifts in the outburst phases with respect to the periastron/apastron passages of the NS is thought to be result of the global perturbations triggered by the truncation of the disk radius. During quiescent state we have not seen any trace of such density wave in the decretion disk that shows itself as the variation of the emission profiles in the spectroscopic data. Wilson et al. (2003) attributed the variations in the $\mathrm{H} \alpha$ emission profile to the existence of the perturbations in the disk and added the difficulties of detection of the density perturbations in XTE J1946+274 since the relatively small viewing angle restricts the size of the projected area to be observed. According to that, only the large-scaled perturbations can be seen in the disk of XTE J1946+274. In contrast to this idea, Silaj et al. (2010) suggested that emission line profile shapes could not be used to estimate the inclination angle of the system since for a given inclination angle different types of profile shapes might be produced as a result of the density changes in the disk-thermal structure. This means that, in the suggested picture of Wilson et al. (2003) for XTE J1946+274, we should have seen the profile changes if there had occurred any density variations in the decretion disk, despite the small inclination angle of the system. Therefore it is very likely not to see any profile changes in the emission lines.

Be stars are variable on all time scales. Short-term variability is commonly associated with either the rotation of the star and/or non-radial pulsations (see e.g. Kızıloğlu et al. 2007; Gutiérrez-Soto et al. 2011), while the long-term variability is believe to be originated by structural changes in the Be decretion disk. The typical time scale for the formation and disintegration of the disk has been found to coincide with the observed time scales of the largest amplitude variations (Lyuty \& Zaitseva 2000; Camero et al. 2014). The observed long optical brightening experienced by XTE J1946+274 may be interpreted as a slow mass ejection event, which started in 2006, peaked in 2010 and rapidly decreased afterwards, reaching a quiescence level in 2012.

The high values obtained for the $\mathrm{H} \alpha \mathrm{EW}$, with the line always seen in emission, may indicate that a large Be circumstellar disk is permanently present in this system. It is also worth noting that although renewed X-ray activity was not observed until 2010, the EW value of the $\mathrm{H} \alpha$ line was already $-37.35 \AA$ in 2007 July (see Table 2). It is possible that the decretion disk of the Be star in XTE J1946+274 is tilted with respect to the orbital motion of the NS. In such a misaligned system warping of the differentially rotating disk can trigger the X-ray activity when the NS capture material from this area (see Okazaki et al. 2013). The $2010 \mathrm{X}$-ray outbursts series emptied accumulated material in the warped regions and the disk dramatically 
decreased around 2011 (see bottom panel of Fig. 7). Although the recovery of the decretion disk was fast, our data point out that the disk reached its maximum size by the time the mass ejection event was over. In such a stable environment the disk did not get warped and therefore no X-rays were detected. This seems to be confirmed by the absence of any variations in our $\mathrm{H} \alpha$ line profiles during the same period.

The ongoing optical/IR brightening, probably initiated around mid 2012, resembles so far the previous mass ejection event between 2006-2012. If the phenomenon repeats we foresee that approximately in two years XTE J1946+274 will manifest in X-rays.

\subsection{NS behavior}

XTE J1946+274 displayed an extended period of activity from 1998 September to 2001 July. Wilson et al. (2003) noted that this period of activity resembled a series of normal outbursts (thirteen) more than a single giant outburst. After approximately $9 \mathrm{yr}$ in quiescence, XTE J1946+274 reawakened in X-rays with a series of five outbursts between 2010 and 2011 (but less intense than in 1998). Once again, two outbursts were observed per orbital period, which did not clearly coincide with the times of periastron and apastron passages of the NS. Fermi/GBM detected pulsations from XTE J1946+274 between 2010 and 2011 (see top panel of Fig. 7).

The last frequency measurement reported by GBM was $0.0635228(3) \mathrm{Hz}\left(P_{\text {spin }}=15.74238(2) \mathrm{s}\right)$ in 2011. Although the frequency histories were not orbitally corrected, and therefore a strong orbital modulation is contaminating the intrinsic torque variations, considering our frequency determination of 2013, the source might have spun down at a rate close to $\dot{v} \sim-0.98 \times 10^{-11} \mathrm{~Hz} \mathrm{~s}^{-1}\left(\dot{P} \sim 2.4 \times 10^{-10} \mathrm{~s} \mathrm{~s}^{-1}\right)$. Spin-up torque/flux correlations for this source were found by Wilson et al. (2003), suggesting the presence of an accretion disk around the NS during X-ray active periods. This scenario is corroborated by Fermi/GBM during the 2010/2011 X-ray outbursts (see Fig. 7).

To determine whether the observed X-ray turn-off was due to centrifugal inhibition of accretion or not (Stella et al. 1986), Wilson et al. (2003) estimated the flux at the onset of this effect by equating the magnetospheric radius and the corotation radius, obtaining a threshold flux for the onset of the centrifugal inhibition of accretion in the range of $0.6-6.0 \times 10^{-11} \mathrm{erg} \mathrm{cm}^{-2} \mathrm{~s}^{-1}$. Our observed flux during quiescence in 2013 was well below this range $\left(\sim 0.1 \times 10^{-11} \mathrm{erg} \mathrm{cm}^{-2} \mathrm{~s}^{-1}\right)$, corresponding to an X-ray luminosity of $\sim 1.2 \times 10^{34} \mathrm{erg} \mathrm{s}^{-1}$ in the $0.3-10 \mathrm{keV}$ range. We would like to point out that the transition flux level depends on several factors (see Eq. (17) in Wilson et al. 2003), although the authors only considered the uncertainty of one of them (the constant factor $k$; see also Eq. (9) in Wilson et al. 2003). To compute a good estimate of the transition flux error it is not an easy task, mainly because there is a considerable uncertainty in the NS parameters. For instance, recent NS mass measurements in X-ray/optical binaries give a range of $M=[1.037,2.44] M_{\odot}$ (mean value $1.568 M_{\odot}$; Lattimer 2012). In addition, Cipolleta et al. (2015) equation of state (EOS) predicts a range of values for the NS radius of 7.64-18.86 km. In particular, Sharmaet al. (2015) found in their model that for a NS of $1.5 M_{\odot}$ the predicted radius should be of the order of $11.67 \mathrm{~km}$. Having this in mind and assuming an error on the NS parameters of about $20 \%$, we would obtain a lower limit for the transitional flux $\sim 0.12 \times 10^{-11} \mathrm{erg}^{-2} \mathrm{~s}^{-1}$, in agreement with the quiescent flux observed by Chandra considering the uncertainties. The flux lower limit would drop with higher errors on the NS parameters. Therefore, the compact object on XTE J1946+274 might have not been in centrifugal inhibition of accretion in 2013 but close to enter in that regimen.

Based on our observation of XTE J1946+274 during quiescence in 2013, and assuming that the source enters the supersonic propeller regime (see e.g. Davies \& Pringle 1981; Henrichs 1983), it is expected to spin down at a rate of $\dot{v}_{\text {super }}=\left(-4 \pi v^{2} \mu^{2}(G M)^{-1} I^{-1}\right)\left(c_{\mathrm{s}} / \mu r_{\mathrm{A}}\right)$, where $\mu$ is the NS magnetic moment, $M$ the mass $\left(1.4 M_{\odot}\right), I$ the moment of inertia, $c_{\mathrm{s}}$ is the sound speed at the magnetospheric radius (which we take here of the order of the free-fall velocity), and $r_{\mathrm{A}}$ is the Alfvén radius (Henrichs 1983). With this $\dot{v}_{\text {super }}$ found to be $\approx-0.13 \times 10^{-12} \mathrm{~Hz} \mathrm{~s}^{-1}$. We note, however, that there is no general consensus on the estimate of the propeller efficiency, and the difference on the estimate of the torque during supersonic propeller state may be as large as $\approx 10^{4}$ under certain conditions (see discussion for $\mathrm{LS} \mathrm{I}+61^{\circ} 303$ in Papitto et al. 2012). It is also possible that other types of torques might be operating simultaneously with the propeller-type torque, e.g. the "frictional" torque (Ghosh 1995, and references therein), with the effect of increasing the observed spin-down torque on the NS. On the other hand, the X-ray luminosity in supersonic propeller regimen is given by $L_{\mathrm{X}}=8 \dot{M} v_{\text {rel }}^{2}$ (Henrichs 1982), where $\dot{M}$ is the mass accretion rate, and $v_{\text {rel }}$ is the relative velocity which is typically of the order of $\sim 1000 \mathrm{~km} \mathrm{~s}^{-1}$. For XTE J1946+274 this yields to $L_{\mathrm{X}} \approx 1.13 \times 10^{34} \mathrm{erg} \mathrm{s}^{-1}$, which is close to what was observed in $2013\left(\sim 1.19 \times 10^{34} \mathrm{erg} \mathrm{s}^{-1}\right)$. Moreover, we found that this is also true for the long quiescence period between 2001 and 2010 . The observed spin-down rate from MJD 55353.01 to 52 115.70, computed using the last frequency determination by Wilson et al. (2003) in $2001(\sim 0.0635 \mathrm{~Hz})$ and the first one detected by GBM $(\sim 0.063421 \mathrm{~Hz})$ in 2010 , gives $\dot{v}_{\mathrm{obs}} \approx 0.18 \times 10^{-12} \mathrm{~Hz} \mathrm{~s}^{-1}$. This result is also close to the observed spin-down rate during this particular period.

\section{Summary and conclusions}

We have performed a long-term multiwavelength study of the Be/X-ray binary system XTE J1946+274, a source which spends almost all its time in quiescence. Our findings include the detection of a large Be circumstellar disk during that phase. We address the absence of major X-ray activity by discussing our results in terms of the neutron star Be-disk interaction.

- A long mass ejection event from the Be-star commenced in 2006, attained its maximum intensity in 2010, and reached quiescence presumably in 2012.

- The high values obtained for the $\mathrm{H} \alpha$ EW (always in emission) point to the permanently presence of a large Be circumstellar disk, probably tilted with respect to the orbital motion of the NS.

- We proposed that the mechanism that might be triggering the $\mathrm{X}$-ray activity is the contact of the NS with the warped area of the Be disk. After the series of X-ray episodes, the warped regions emptied and the disk dramatically decreased.

- There was an absence of variations in the $\mathrm{H} \alpha$ line profiles during the posterior fast recovery of the disk. This took place in parallel to the decay of the optical mass ejection event. In a much more stable environment the disk did not get warped and therefore no X-rays were detected.

- On the other hand, pulsations from the NS have been detected during X-ray quiescence in 2013, with $P_{\text {pulse }}=$ $15.757(1) \mathrm{s}$ and an rms pulse fraction of $32.1(3) \%$. 
- The 0.3-10 keV X-ray spectrum of the NS was well fitted with either an absorbed black-body or absorbed power-law models $(k T=1.43 \pm 0.17$ and $\Gamma=0.9 \pm 0.4)$.

- The observed X-ray luminosity during quiescence is close to that observed when the NS is in supersonic propeller regimen.

- The ongoing optical/IR brightening resembles so far the previous mass ejection event. If the proposed scenario from above is valid we predict that approximately in two years XTE J1946+274 will show major X-ray activity.

Acknowledgements. We thank the anonymous referee for his/her helpful comments and suggestions. This article is partially based on service observations made with the IAC80 and TCS telescopes operated on the island of Tenerife by the Instituto de Astrofísica de Canarias (IAC) in the Spanish Observatorio del Teide. The present work is also based on observations made with the Nordic Optical Telescope, operated by the Nordic Optical Telescope Scientific Association at the Observatorio del Roque de los Muchachos (IAC), La Palma, Spain. The Albireo spectrograph at the $1.5-\mathrm{m}$ telescope is operated by the Instituto de Astrofísica de Andalucía at the Sierra Nevada Observatory. We thank TÜBITAK and ROTSE collaboration for partial support in using the RTT 150 and ROTSEIIId Telescopes with project numbers TUG-RTT150.08.45, 12ARTT150-264-1 and ROTSE-40. The scientific results reported in this article are based in part on data obtained from the Chandra Data Archive. The work of J.G.S. is supported by the Spanish Programa Nacional de Astronomía y Astrofísica under contract AYA2012-39246-C02-01. E.N. acknowledges a VALi+d postdoctoral grant from the Generalitat Valenciana and was supported by the Spanish Ministry of Economy and Competitiveness under contract AYA 2010-18352. A.C. was supported by the AYA2012-39303, SGR2009-811 and iLINK2011-0303 grants. M.Ö. A. acknowledges support from TÜBİTAK, The Scientific and Technological Research Council of Turkey, through the research project 106T040. We thank the anonymous refree for his/her helplful comments dans suggestions.

\section{References}

Akerlof, C. W., Kehoe, R. L., McKay, T. A., et al. 2003, PASP, 115, 132 Anders, E., \& Grevesse, N. 1989, Geochim. Cosmochim. Acta, 53, 197

Arnaud, K. A. 1996, in Astronomical Data Analysis Software and Systems V, eds. G. H. Jacoby \& J. Barnes, ASP Conf. Ser., 101, 17

Balucinska-Church, M., \& McCammon, D. 1992, ApJ, 400, 699

Bertin, E., \& Arnouts, S. 1996, A\&AS, 117, 393

Caballero, I., Pottschmidt, K., Bozzo, E., et al. 2010, ATel, 2692, 1
Camero, A., Zurita, C., Gutierrez Soto, J., et al. 2014, A\&A, 568, A115

Camero-Arranz, A., Finger, M. H., Ikhsanov, N. R., Wilson-Hodge, C. A., \& Beklen, E. 2010a, ApJ, 708, 1500

Camero-Arranz, A., Finger, M. H., Wilson-Hodge, C., \& Jenke, P. 2010b, ATel, 2677, 1

Campana, S., Israel, G., \& Stella, L. 1999, A\&A, 352, L91

Cipolleta, F., Cherubini, C., Filippi, S., Rueda, J. A., Ruffini, R. 2015, Phys. Rev. D 92, 023007

Davies, R. E., \& Pringle, J. E. 1981, MNRAS, 196, 209

Finger, M. H., Beklen, E., Narayana Bhat, P., et al. 2009, Fermi Symp., eConf Proc. C091122

Ghosh, P. 1995, JA\&A, 16, 289

Gutiérrez-Soto, J., Reig, P., Fabregat, J., \& Fox-Machado, L. 2011, in IAU Symp. 272, I eds. C. Neiner, G. Wade, G. Meynet, \& G. Peters, 505

Hanuschik, R. W. 1989, Ap\&SS, 161, 61

Heindl, W. A., Coburn, W., Gruber, D. E., et al. 2001, ApJ, 563, L35

Henrichs, H. F. 1982, Ph.D. Thesis, University of Amsterdam

Henrichs, H. F. 1983, in Accretion-Driven Stellar X-ray Sources, eds. W. H. G. Lewin, \& E. P. J. van den Heuvel, 393

Illarionov, A. F., \& Kompaneets, D. A. 1990, MNRAS, 247, 219

Kızıloğlu, Ü., Kızıloğlu, N., \& Baykal, A. 2005, AJ, 130, 2766

Kızıloğlu, U., Kızıloğlu, N., Baykal, A., Yerli, S. K., \& Özbey, M. 2007, A\&A, 470,1023

Krimm, H. A., Barthelmy, S. D., Baumgartner, W., et al. 2010, ATel, 2663, 1

Krimm, H. A., Holland, S. T., Corbet, R. H. D., et al. 2013, ApJS, 209, 14

Lattimer, J. M., 2012, ARNPS, 62, 485

Lenz, P., \& Breger, M. 2005, Comm. Asteroseism., 146, 53

Lyuty, V. M., \& Zaitseva, G. V. 2000, VizieR Online Data Catalog: J/PAZh/26/13

Müller, S., Kühnel, M., Caballero, I., et al. 2012, A\&A, 546, A125

Müller, S., Kühnel, M., Pottschmidt, K., et al. 2010, ATel, 3077, 1

Nakajima, M., Mihara, T., Nakagawa, Y. E., et al. 2010, ATel, 3048, 1

Okazaki, A. T., Hayasaki, K., \& Moritani, Y. 2013, PASJ, 65, 41

Papitto, A., Torres, D. F., \& Rea, N. 2012, ApJ, 756, 188

Priedhorsky, W. C., \& Holt, S. S. 1987, Space Sci. Rev., 45, 291

Roberts, D. H., Lehar, J., \& Dreher, J. W. 1987, AJ, 93, 968

Scargle, J. D. 1982, ApJ, 263, 835

Sharma, B. K., Centelles, M., Vinas, X., Baldo, M., Burgio, G. F. 2015, A\&A, in press, DOI: 10.1051/0004-6361/201526642

Silaj, J., Jones, C. E., Tycner, C., Sigut, T. A. A., \& Smith, A. D. 2010, ApJS, 187,228

Smith, D. A., \& Takeshima, T. 1998, ATel, 36, 1

Stella, L., White, N. E., \& Rosner, R. 1986, ApJ, 308, 669

Verrecchia, F., Israel, G. L., Negueruela, I., et al. 2002, A\&A, 393, 983

Wilson, C. A., Finger, M. H., Wilson, R. B., \& Scott, D. M. 1998, IAU Circ., 7014, 2

Wilson, C. A., Finger, M. H., Coe, M. J., \& Negueruela, I. 2003, ApJ, 584, 996 